\title{
Shape and Reinforcement Optimization of Underground Tunnels*
}

\author{
Kazem GHABRAIE**, Yi Min XIE ${ }^{* * *}$, Xiaodong HUANG ${ }^{* * *}$ \\ and Gang REN ${ }^{* * *}$ \\ ** Faculty of Engineering and Surveying, University of Southern Queensland \\ West Street, Toowoomba, QLD 4350, Australia \\ E-mail: kazem.ghabraie@usq.edu.au \\ *** School of Civil, Environmental and Chemical Engineering, RMIT University \\ GPO Box 2476V, Melbourne, VIC 3001, Australia
}

\begin{abstract}
Design of support system and selecting an optimum shape for the opening are two important steps in designing excavations in rock masses. Currently selecting the shape and support design are mainly based on designer's judgment and experience. Both of these problems can be viewed as material distribution problems where one needs to find the optimum distribution of a material in a domain. Topology optimization techniques have proved to be useful in solving these kinds of problems in structural design. Recently the application of topology optimization techniques in reinforcement design around underground excavations has been studied by some researchers. In this paper a three-phase material model will be introduced changing between normal rock, reinforced rock, and void. Using such a material model both problems of shape and reinforcement design can be solved together. A well-known topology optimization technique used in structural design is bi-directional evolutionary structural optimization (BESO). In this paper the BESO technique has been extended to simultaneously optimize the shape of the opening and the distribution of reinforcements. Validity and capability of the proposed approach have been investigated through some examples.
\end{abstract}

Key words : Underground Excavation, Tunnel Reinforcement, Topology Optimization, Shape Optimization, Finite Element Analysis

\section{Introduction}

During the last two decades topology optimization has attracted considerable attention and the techniques in this field have been improved significantly. Several physical problems have been tackled in this context. However the application of topology optimization tools in geotechnical problems has not been studied thoroughly. In spite of the great potential in this class of problems there are only a few published works in this area. Among these works some attempted to optimize the shape of underground openings ${ }^{(1),(2)}$ while others tried to optimize the topology of reinforcement around a tunnel with a predefined shape ${ }^{(3)-(6)}$. In this paper, attempt is made to optimize the shape of the opening and the topology of the surrounding reinforcement simultaneously. It will be shown that the sensitivities of these two optimization problems only differ in constant values. Hence the two optimization problems can be solved together with almost no extra computational effort.

The topology optimization techniques used in aforementioned works are the Solid Isotropic Material with Penalization (SIMP) method, the Evolutionary Structural Optimization (ESO) technique and its Bi-directional version, the BESO method. These methods are the most popular topology optimization techniques due to their ease of use and reasonable results. All these methods control the topology using a material distribution approach, that is, they change the topology by adding and removing material to and from the design domain.

*Received 20 Nov., 2009 (No. 09-0708) [DOI: 10.1299/jcst.4.51]

Copyright (c) 2010 by JSME 
Originally proposed by Bendsøe in $1989^{(7)}$, the SIMP method is based on the homogenization technique which was introduced earlier by Bendsøe and Kikuchi ${ }^{(8)}$. The SIMP material model assumes that the Young's modulus of the designing material is a function of the material's relative density $(E=E(\rho))$. The relative density function varies between a very small positive value $\rho_{\text {min }}$ (representing voids) and 1 (representing solids). These relative densities are treated as design variables and the SIMP method adjusts them iteratively using a gradient-based update scheme to achieve the optimum of the chosen objective function ${ }^{(7)}$. The relation between the relative density and the modulus of elasticity in the SIMP method is a power-law relationship defined as $E=\rho^{p} E_{0}$ where $p$ is a penalty factor and $E_{0}$ is the modulus of elasticity of the base material ${ }^{(7),(9)}$. The penalty factor penalizes the intermediate values of the relative densities and pushes the final topology towards a binary solid/empty topology. Bigger penalties result in more clear topologies but they will cause some convergency problems. On the other hand small penalties will result in blurred topologies with lots of elements having intermediate (virtual) density values. Even with large penalty factors the final result will still have some elements with intermediate densities.

The BESO method was proposed in late $90 \mathrm{~s}^{(10),(11)}$ as an improved version of the ESO method which was originally introduced in early 90 s by Xie and Steven ${ }^{(12),(13)}$. The ESO method improves the design by gradually removing the inefficient elements. In the BESO method, on the other hand, a bi-directional evolutionary strategy is applied which also allows the strengthening of the efficient parts by adding material. The efficiency of elements can be calculated by sensitivity analysis of the considered objective function or can be assigned intuitively ${ }^{(14)}$.

The main difference between the SIMP and the BESO methods is in the nature of the design variables they use. While the SIMP material model uses continuous design variables, the BESO method uses discrete values. This property of the BESO technique assures that the results are always clear because no intermediate density is in use. The clearness of the results is important in shape optimization since the boundaries in a blurred image are not easily definable. For this reason the BESO technique is easier to implement for shape optimization of the opening. In this paper the BESO method is used for solving both problems of shape optimization of the opening and topology optimization of reinforcements. These two problems can both be modeled as two-phase material distribution problems. For shape optimization the material is changing between solids and voids. In reinforcement optimization, on the other hand, the material can be switched between original rock and reinforced rock.

In the original BESO, unlike the SIMP material model, elements can be removed completely. Topology optimization methods which remove elements completely are sometimes referred to as hard kill methods as oppose to soft kill methods like the SIMP method. In hard kill methods only the non-void elements will remain in the mesh and so the finite element analysis can be performed faster. However in hard kill methods the sensitivity of void elements cannot be calculated directly from the analysis results and should be extrapolated from the surrounding solid elements. In this paper a soft kill BESO has been adopted where the void elements are represented by a very soft material. In this manner the sensitivities of voids are directly calculable.

\section{Material Model}

Although the geomechanical materials are naturally inhomogeneous, non-linear, anisotropic, and inelastic ${ }^{(15)}$, in excavation design in rocks, modeling them as an isotropic, homogeneous material and assuming linear elastic behavior can be instructive and sometimes can predict the real behavior with acceptable accuracy ${ }^{(16)}$. In fact the simplified linear elastic material model is still the most common material model used in geomechanics ${ }^{(15)}$. Moreover the results of a linear analysis can be used as a first-order approximation of non-linear cases. Most of previous works ${ }^{(1),(3)-(6)}$ which applied the topology optimization techniques in excavation design, have adopted the linear elastic material model. As the application of topology 
optimization techniques in excavation design has not been investigated thoroughly and in order to produce comparable results with previous works and verifying the current approach, the linear elastic material model has been used here. The authors are currently working on implementing elasto-plastic material models.

The homogeneity assumption is valid in case of intact rock and highly weathered rock mass. In case of massive rocks with few major discontinuities, the overall behavior of the rock mass is predominantly influenced by the discontinuities. Hence, in these types of rocks, the homogeneity assumption is far from reality. Reinforcement optimization of underground excavations in such types of rocks is addressed in Ref. (17).

In underground excavation it rarely happens that the ground material can adequately resist the consequences of stress relief. The use of supports is thus usually unavoidable. Active support design is based on the idea that the soil or rock mass is actively contributing in carrying the load caused by excavation ${ }^{(4)}$. One way of such supporting design is using rock bolts. Using rock bolt systems, the rock mass can effectively be reinforced only where it is not strong enough. Homogenized properties can be used to model the behavior of the reinforced parts of rock mass ${ }^{(18)}$. In this paper, in line with previous publications ${ }^{(3)}{ }^{(6)}$, a linear elastic behavior is assumed for reinforced rock. Further discussion on validity of this type of analysis can be found in Ref. (4). The moduli of elasticity of host rock and reinforced rock are represented by $E^{O}$ and $E^{R}$ respectively. As mentioned before, the void areas are considered to be made of a very weak material. The modulus of elasticity of this weak material is represented by $E^{V}$ and it is assumed that $E^{V}=0.001 E^{O}$. It is also assumed that all these materials have same Poisson's ratio equal to 0.3 .

\section{Objective Function and Problem Statement}

Consider a simple design case depicted in Fig. 1. In this figure, $\Gamma$ represents the boundary of the opening. The minimum dimensions shown in the figure can be due to some design restrictions. The placement, orientation and the length of rock bolts has been depicted by solid line segments in this figure. The dark shaded area $\Omega$ with the outer boundary of $\partial \Omega$ and inner boundary of $\Gamma$ is the reinforced area of the design. Having found this reinforced area one can choose the proper location and length of the reinforcing bars and vice versa. In the shape optimization of the opening one deals with finding $\Gamma$ while in reinforcement optimization the shape and topology of $\partial \Omega$ and $\Omega$ are of interest. The simultaneous shape and reinforcement optimization can be viewed as finding the optimal $\Omega$ when both its inner and outer boundaries $\Gamma$ and $\partial \Omega$ are changing.

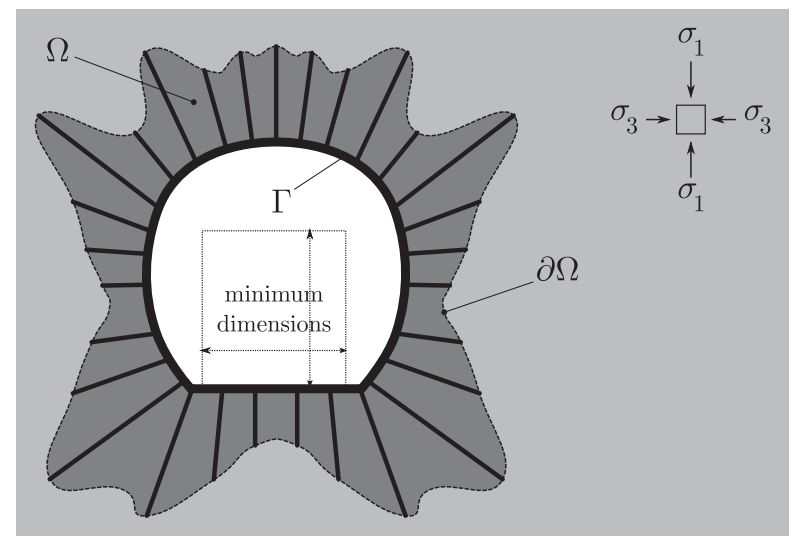

Fig. 1 A simple design case.

Ren et al. ${ }^{(1)}$ and Ghabraie et al. ${ }^{(2)}$ have used a full stress design strategy to optimize the shape of the underground opening. In full stress design strategy, it is (intuitively) assumed that in the optimal design all parts of the structure are fully stressed. However, in spite of reasonable results, this approach is based on intuition lacking a mathematically proven basis. 
On the other hand calculating sensitivities for stress based objective functions is not an easy task. In this paper the mean compliance has been used as objective function which is the most common objective function used in topology optimization problems. Considering a volume constraint on reinforcement material and restricting the size of the opening, the problem of concern can be expressed as

$$
\begin{array}{lc}
\min _{x_{1}, x_{2}, \ldots, x_{n}} & c=\mathbf{f}^{T} \mathbf{u} \\
\text { such that } & V_{R} \leq \bar{V}_{R}, \\
& V_{V}=\bar{V}_{V}
\end{array}
$$

where $x_{1}, x_{2}, \ldots, x_{n}$ are design variables, $c$ is the mean compliance, $\mathbf{f}$ is the nodal force vector, $\mathbf{u}$ stands for nodal displacement vector, and $V_{R}, V_{V}, \bar{V}_{R}$ and $\bar{V}_{V}$ are reinforcement and void volume and their corresponding limits respectively. It can be seen from Eq. (1) that the mean compliance is equivalent to twice of the strain energy. Minimizing compliance will be equivalent to maximizing the stiffness of structure. The problem thus will be finding the stiffest design with prescribed opening size and predefined upper limit of the volume of reinforcement material.

Because the mean compliance is a convex and self-adjoint function its sensitivity analysis is relatively easy and computationally efficient ${ }^{(19)}$. For problems with constant load (where load is not a function of design variables) sensitivities of mean compliance can be easily calculated via adjoint method $^{(19)}$ or direct differentiation $^{(20)}$ as

$$
\frac{\partial c}{\partial x_{i}}=-\mathbf{u}^{T} \frac{\partial \mathbf{K}}{\partial x_{i}} \mathbf{u}
$$

where $\mathbf{K}$ stands for stiffness matrix and $x_{i}$ is the $i$-th design variable. The stiffness matrix in element level can be related to design variables by

$$
\mathbf{K}_{i}\left(x_{i}\right)=\frac{E\left(x_{i}\right)}{E^{O}} \mathbf{K}_{i}^{O}
$$

where $E$ is the elasticity modulus of the element $i$ which is a function of the element's design variable. $\mathbf{K}_{i}^{O}$ is the stiffness matrix of element $i$ as if it was made of original rock, that is

$$
\mathbf{K}_{i}^{O}=\int_{V_{i}} \mathbf{B}_{i}^{T} \mathbf{D}^{O} \mathbf{B}_{i} \mathrm{~d} V
$$

where $\mathbf{D}^{O}$ represents the constitutive matrix for original rock, $\mathbf{B}_{i}$ represents the straindisplacement matrix and $V_{i}$ is the volume of element $i$.

In order to maintain the topology of the hole for shape optimization, the boundary of the hole should be determined and only the boundary elements should be allowed to change. In this paper it is assumed that there is a shotcrete lining around the opening with material properties similar to that of reinforced rock. In this manner, in the shape optimization of the opening, the material can be changed from void to reinforced rock and vice versa. In the reinforcement optimization, on the other hand the two material phases are original rock and reinforced rock.

\section{Material Interpolation Scheme}

For a general two-phase material case, the interpolated modulus of elasticity can be defined as

$$
E(x)=E_{1}+x\left(E_{2}-E_{1}\right)
$$

where $E_{1}$ and $E_{2}$ are Young's moduli of the two materials. Using Eq. (5) in Eq. (2), the latter can be rewritten as

$$
\frac{\partial c}{\partial x_{i}}=-\frac{E_{2}-E_{1}}{E^{O}} \mathbf{u}_{i}^{T} \mathbf{K}_{i}^{O} \mathbf{u}_{i}
$$


where $\mathbf{u}_{i}$ indicates local nodal displacements at the element level for the $i$-th element. The change in objective function due to a change in an element can be approximated as

$$
\Delta c=\Delta x_{i} \frac{\partial c}{\partial x_{i}}
$$

If the material of an element changes, one can calculate the approximate change in objective function by substituting corresponding $\Delta x_{i}$ value in Eq. (7).

\subsection{Shape optimization of the tunnel}

In shape optimization of the opening the two phases of the material are void and reinforced rock so Eqs. (5) and (7) can be rewritten as

$$
E(x)=E^{V}+x\left(E^{R}-E^{V}\right)
$$

and

$$
\Delta c=-\Delta x_{i} \cdot \frac{E^{R}-E^{V}}{E^{O}} \mathbf{u}_{i}^{T} \mathbf{K}_{i}^{O} \mathbf{u}_{i}
$$

respectively. Note that in Eq. (8) the void and the reinforced rock phases are represented by values of 0 and 1 for $x$ respectively. Now for an element changing from void to reinforced rock $\left(\Delta x_{i}=x^{R}-x^{V}=+1\right)$ one can write

$$
\Delta c=-\frac{E^{R}-E^{V}}{E^{V}} \mathbf{u}_{i}^{T} \mathbf{K}_{i} \mathbf{u}_{i}, \quad i \in \mathcal{V}
$$

with $\mathcal{V}$ standing for the set of the numbers of currently void elements. Note that in Eq. (10) the $i$-th element is void so $\mathbf{K}_{i}=\mathbf{K}_{i}^{V}$.

In the same way when an element switches from reinforced rock to void, the change in objective function can be approximated as

$$
\Delta c=\frac{E^{R}-E^{V}}{E^{R}} \mathbf{u}_{i}^{T} \mathbf{K}_{i} \mathbf{u}_{i}, \quad i \in \mathcal{R}
$$

Here $\mathcal{R}$ is the set of the numbers of currently reinforced elements. The positive sign in Eq. (11) shows that changing a reinforced element to a void will increase compliance (decrease stiffness) while the negative sign in Eq. (10) states that replacing a void element with a reinforced one will make the structure stiffer.

Based on Eqs. (10) and (11) one can define the following sensitivity number for shape optimization of the opening

$$
\alpha_{S}= \begin{cases}E^{V}\left(E^{R}-E^{V}\right) \mathbf{u}_{i}^{T} \mathbf{K}_{i} \mathbf{u}_{i}, & i \in \mathcal{V} \\ E^{R}\left(E^{R}-E^{V}\right) \mathbf{u}_{i}^{T} \mathbf{K}_{i} \mathbf{u}_{i}, & i \in \mathcal{R}\end{cases}
$$

Here the sensitivity number is defined as the change in compliance multiplied by the square of the Young's modulus. This definition prevents infinite sensitivity numbers for the case of $E^{V}=0$. Furthermore in this way, the stiffer materials get higher sensitivity numbers than the softer materials.

Considering the sensitivity numbers defined in Eq. (12) the reinforced elements with the lowest sensitivity numbers are the least efficient elements and should be change to voids while the void elements with the highest sensitivity numbers are the most efficient ones and should be switched to reinforced rock.

\subsection{Reinforcement optimization}

In topology optimization of reinforcements, original rock elements can be turned into reinforced elements and vice versa. The interpolation equation Eq. (5) can hence be written as

$$
E(x)=E^{O}+x\left(E^{R}-E^{V}\right)
$$


This case is completely similar to that of shape optimization. The only difference is that $E^{V}$ is replaced by $E^{O}$ here. Similar to the shape optimization case, a change in the objective function due to reinforcing an element can be calculated as

$$
\Delta c=-\frac{E^{R}-E^{O}}{E^{O}} \mathbf{u}_{i}^{T} \mathbf{K}_{i} \mathbf{u}_{i}, \quad i \in O
$$

where $O$ is the set of the original rock elements' numbers. On the other hand if an element changes from reinforced rock to original rock the change in objective function will be

$$
\Delta c=\frac{E^{R}-E^{O}}{E^{R}} \mathbf{u}_{i}^{T} \mathbf{K}_{i} \mathbf{u}_{i}, \quad i \in \mathcal{R}
$$

Based on Eqs. (14) and (15) the following sensitivity number can be defined for the reinforcements optimization

$$
\alpha_{R}= \begin{cases}E^{O}\left(E^{R}-E^{O}\right) \mathbf{u}_{i}^{T} \mathbf{K}_{i} \mathbf{u}_{i}, & i \in O \\ E^{R}\left(E^{R}-E^{O}\right) \mathbf{u}_{i}^{T} \mathbf{K}_{i} \mathbf{u}_{i}, & i \in \mathcal{R}\end{cases}
$$

The reinforced elements with the lowest sensitivity numbers are the least efficient elements and should be changed to original rock. On the other hand the rock elements with the highest sensitivity numbers are the most efficient ones and should be changed to reinforced rock.

Sensitivity numbers defined in Eqs. (12) and (16) only differ in constant coefficients and both can be calculated by multiplying the strain energy of the elements by the calculated coefficients. That means the computational time to solve these two problems is nearly same as that of a single problem.

\section{FILTERING SENSITIVITIES}

It is known that some topology optimization methods including the BESO and the SIMP methods are prone to numerical instabilities like the formation of checkerboard patterns and mesh dependency ${ }^{(22)}$. One of the simplest approaches which is known to be capable of overcoming these two instabilities is filtering the sensitivities ${ }^{(22)-(24)}$. In filtering technique a new sensitivity number will be calculated based on the sensitivity numbers of the element itself and its neighboring elements. The following filtering scheme has been used in this paper to calculate the filtered sensitivity numbers

$$
\hat{\alpha}_{i}=\frac{\sum_{j=1}^{n} \alpha_{j} H_{i j}}{\sum_{j=1}^{n} H_{i j}}
$$

where $\hat{\alpha}_{i}$ is the filtered sensitivity number of the $i$-th element, $n$ is the number of elements and

$$
H_{i j}=\max \left\{0, r_{f}-d_{i j}\right\}
$$

Here $r_{f}$ is the filtering radius and $d_{i j}$ is the distance between the centers of the $i$-th and the $j$-th elements. Note that the equation Eq. (17) is actually a weighted average which results in greater values in elements near the areas of high sensitivity and vice versa. Using this filtering scheme will result in smoother results. The smoothness of the final result increases by using larger filtering radii. However one should note that choosing a very large filtering radius can result in convergency problems and sub-optimal solutions.

\section{BESO PROCEDURE}

The BESO procedure iteratively switches elements between different materials (and voids) based on their sensitivity numbers. If in the initial design the materials' volumes are not within the constraints in Eq. (1), then these volumes will be adjusted gradually to meet the constraints. This can be achieved by controlling the number of switches between different materials. In Ref. (24) an algorithm has been proposed for gradually adjusting the materials' volumes. If one starts from a feasible design there is no need to change the volume. In this case the number of elements to be added or removed should be equal to keep the volume constant. 
In the examples solved here a feasible initial design is used. At every iteration a number of elements will switch between reinforcements and voids to optimize the shape of the opening based on Eq. (12). Then some other switches will be applied between normal and reinforced rocks to optimize the topology of reinforcement's distribution based on Eq. (16). Restricting the program to switch only a few elements each time one can prevent sudden changes to the design. The maximum number of switches between different elements at each iteration is referred to as move limit. Using larger move limits one can obtain faster convergence but may lose some optimum points. With a small move limit, the evolution of the objective function should show a relatively monotonic trend with a steep descent at initial iterations reaching a flat line at the end showing convergency. Getting such evolution trend one can ensure that the optimization procedure is working well.

To keep up with the shotcrete lining the elements on the boundary of the hole should be changed to shotcrete elements after each update in the hole's shape. Therefore the number of shotcrete elements might change during optimization while the total value of reinforced rock and shotcrete is constrained. In order to satisfy this volume constraint, in reinforcement optimization the number of reinforcing and weakening elements should be adjusted.

The algorithm of the BESO procedure used here is briefly reviewed in Fig. 2.

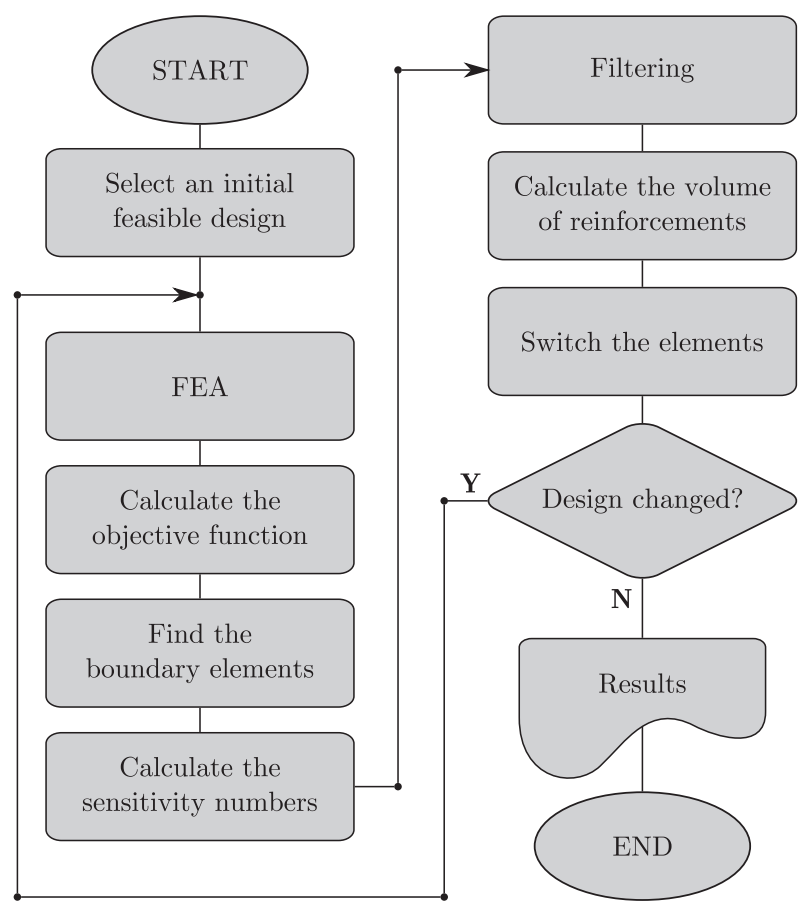

Fig. 2 The flowchart of the BESO procedure for optimizing the shape and the reinforcement of tunnels.

\section{Examples}

For verification purpose the proposed BESO algorithm has been used to solve some examples. In these examples the relative values of moduli of elasticity of reinforced rock, original rock, and void elements have been considered as 10000:3000:3 respectively. In all cases it is assumed that the tunnel is long and straight enough to validate plane strain assumption. The semi-infinite underground domain has been modeled by a large finite element mesh. In all examples the outer boundaries of the design domain have been considered as non-designable rock elements in order to prevent reinforcing of far fields. Because the discretized domain is very large in compare to the size of the opening, changes in the opening's shape will not have a considerable effect on the overall compliance. The objective function is thus limited to the compliance of designable domain only. The filtering radius is considered equal to twice of the elements' size. The move limit has been limited to five elements. In all examples it is 
assumed that the tunnel should have a flat floor. To fulfill this requirement in all examples a layer of non-designable reinforced rock has been considered at the bottom of the opening.

\subsection{Example 1}

In the first example, a single tunnel under biaxial stress like the one sckeched in Fig. 1 has been considered. An initial guess design together with non-designable elements has been depicted in Fig. 3. The minimum size of the opening is $W=2.4 \mathrm{~m}$ and $H=1.6 \mathrm{~m}$. This area is restricted to void elements by setting a rectangular area of non-designable voids. The size of the opening is $7.92 \mathrm{~m}^{2}$. The upper limit for the volume of the reinforcement material is chosen equal to $14.8 \mathrm{~m}^{2}$. The infinite domain has been replaced by a large finite domain of size $20 \mathrm{~m} \times 20 \mathrm{~m}$ surrounding the opening. To model the stress conditions uniform distributed loads with consistent magnitudes have been applied on top, right and left sides and the bottom is restrained against vertical displacement (Fig. 3).

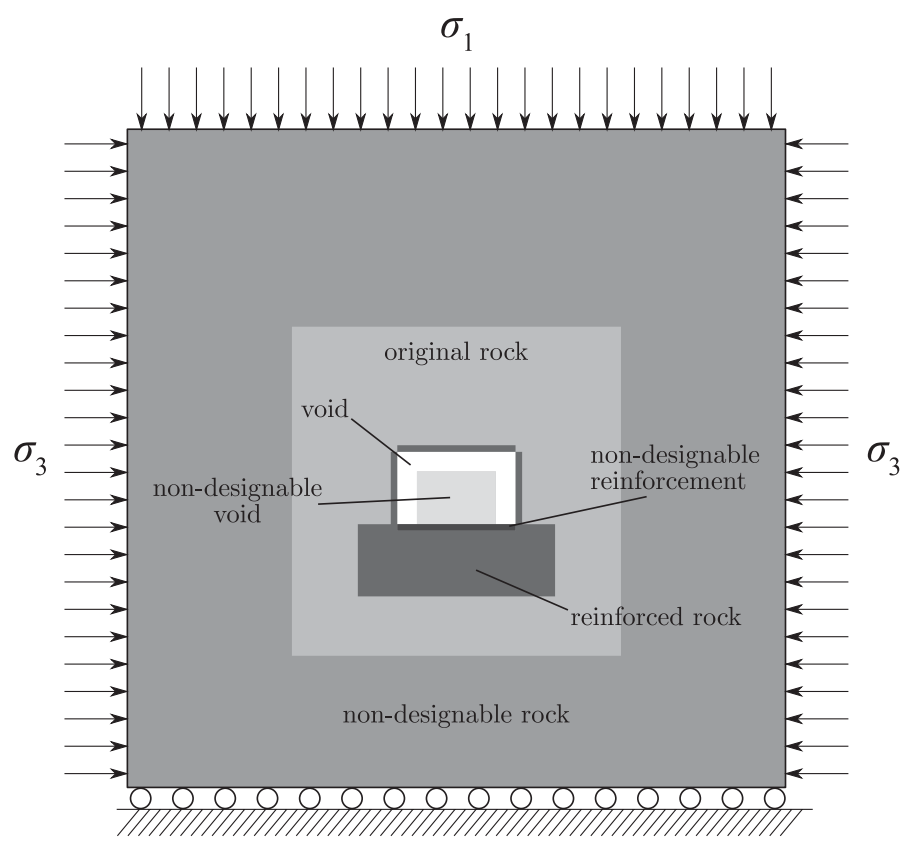

Fig. 3 An initial guess design illustrating the design domain, non-designable elements, loading, and restraints.

Because of symmetry only half of the design domain has been considered in finite element analysis with proper symmetry constraints. A typical $2 \mathrm{D}$ mesh consisting of $50 \times 100$ equally sized quadrilateral 4-node elements has been used to discretize the half model.

Three cases with different values of horizontal to vertical stress ratio $(\lambda)$ has been considered. Figure 4 show the final results together with corresponding objective function evolution for $\lambda=0.4, \lambda=0.7$, and $\lambda=1.2$. As shown in these figures in all cases the objective function changes almost monotonically and smoothly. It can be seen that the final shape of the opening and the final topology of reinforcements change dramatically with load ratio. The aspect ratio of the optimum opening shapes show a correlation with the applied load ratios which is also reported in Ref.(1) and (2). The initial and the final values of the objective function are reported in Table 1.

After obtaining the results, the boundaries of the opening can be smoothed using a postprocessing subroutine. A smoothing procedure based on Bézier curves has been applied in this paper. After obtaining the final shapes, the boundaries between different materials are extracted. The boundary lines are then smoothed using Bézier curves. Finally the location of smoothed nodes are used to produce a new mesh. The smoothed results of this example are shown in Fig. 5. Knowing the reinforced area one can choose the location and length of the rockbolts and the thickness of shotcrete. 

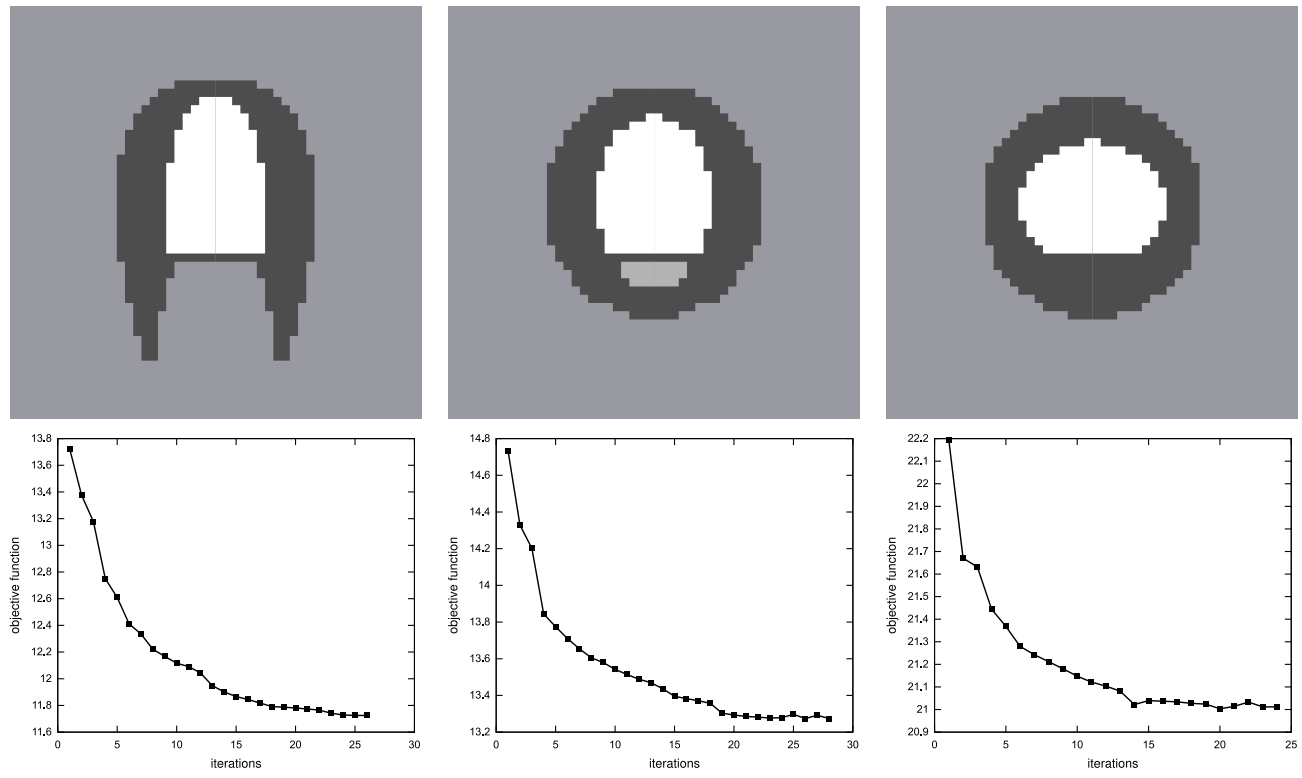

Fig. 4 The final topology and the evolution of the value of the objective function for load ratios of 0.4 (left), 0.7 (middle) and 1.2 (right) in example 1.

Table 1 The initial and final objective function's values for three cases in example 1 .

\begin{tabular}{c|ccc}
\hline Case & Initial value & Final value & Improvement(\%) \\
\hline$\lambda=0.4$ & 13.72 & 11.72 & 14.57 \\
$\lambda=0.7$ & 14.73 & 13.27 & 9.90 \\
$\lambda=1.2$ & 22.19 & 21.01 & 5.33 \\
\hline
\end{tabular}
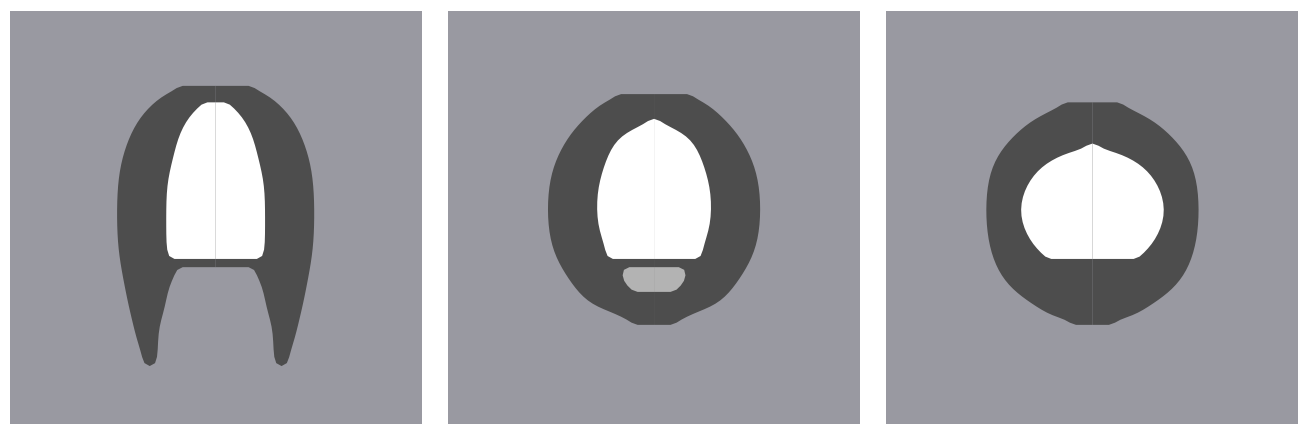

Fig. 5 The smoothed results of the first example. 


\subsection{Example 2}

In the second example a distributed traffic load is applied on the tunnel's floor over the width of non-designable void elements $(2.4 \mathrm{~m})$. The magnitude of this traffic load has been considered as $\sigma_{\text {traffic }}=0.25 \sigma_{1}$. The smoothed final results for the three load cases are illustrated in Fig. 6. As expected the reinforcement topology has changed in compare to the results obtained in previous example. Especially for the case of $\lambda=0.7$ the unreinforced area under the tunnel's floor in previous example has been replaced by a reinforced bar. The optimum shapes of the hole have also changed in compare to the first example. It can be seen that the optimal tunnels have wider floors and are shorter than the shapes obtained in the first example.


Fig. 6 The smoothed optimum results for problems with traffic load and load ratios of 0.4 (left), 0.7 (middle), and 1.2 (right) in example 2.

\subsection{Example 3}

Figure 7 shows a sketch of the loading and design restrictions for two identical parallel tunnels. The minimum allowable dimensions of the opening is restricted to the width of $W$ and height of $H$. It is also required that the tunnel has a flat floor. The area of the opening should be fixed to $A$. The tunnel is under biaxial stress state with the horizontal to vertical stress ratio of $\lambda=\sigma_{3} / \sigma_{1}$. The traffic loads of trains can be considered as a uniform distributed load over the width $W$.

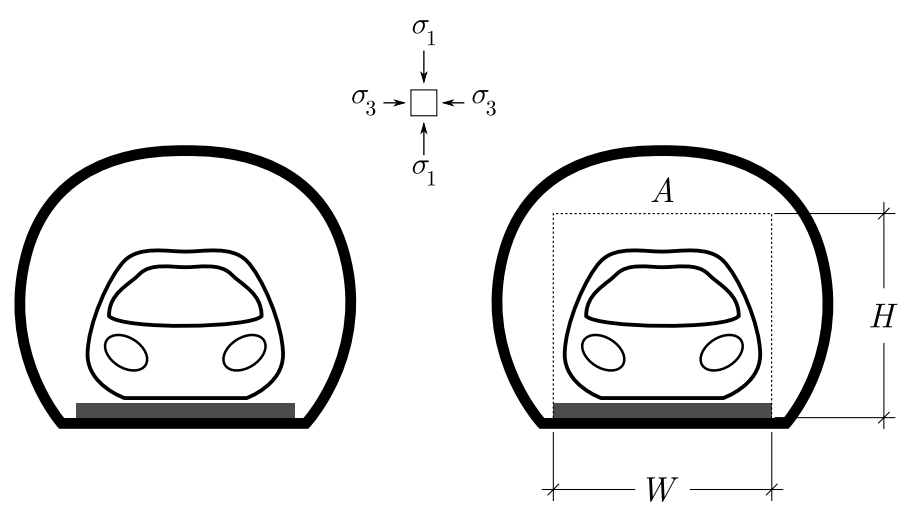

Fig. 7 A simple sketch of the loading and design restrictions of two parallel tunnels.

The initial guess design and the design domain have been illustrated in Fig. 8. It is assumed that the minimum width and height of the opening are $W=2.4 \mathrm{~m}$ and $H=2.8 \mathrm{~m}$. The total area of the opening is restricted to $A=16 \mathrm{~m}^{2}$. The magnitude of the traffic load is considered equal to $\sigma_{\text {traffic }}=0.25 \sigma_{1}$. The semi-infinite domain is replaced by a $40 \mathrm{~m} \times 40 \mathrm{~m}$ area. An area of $20 \mathrm{~m} \times 20 \mathrm{~m}$ surrounding the tunnels has been selected as the design domain. The upper limit for the volume of the reinforcement material is considered as $58.88 \mathrm{~m}^{2}$. Again because of symmetry only half of the design domain has been considered in the FEA.

The optimum designs obtained for the three load ratios of $0.4,0.7$ and 1.2 are depicted in Fig. 9. It can be seen that by increasing horizontal pressure the reinforcements around the 


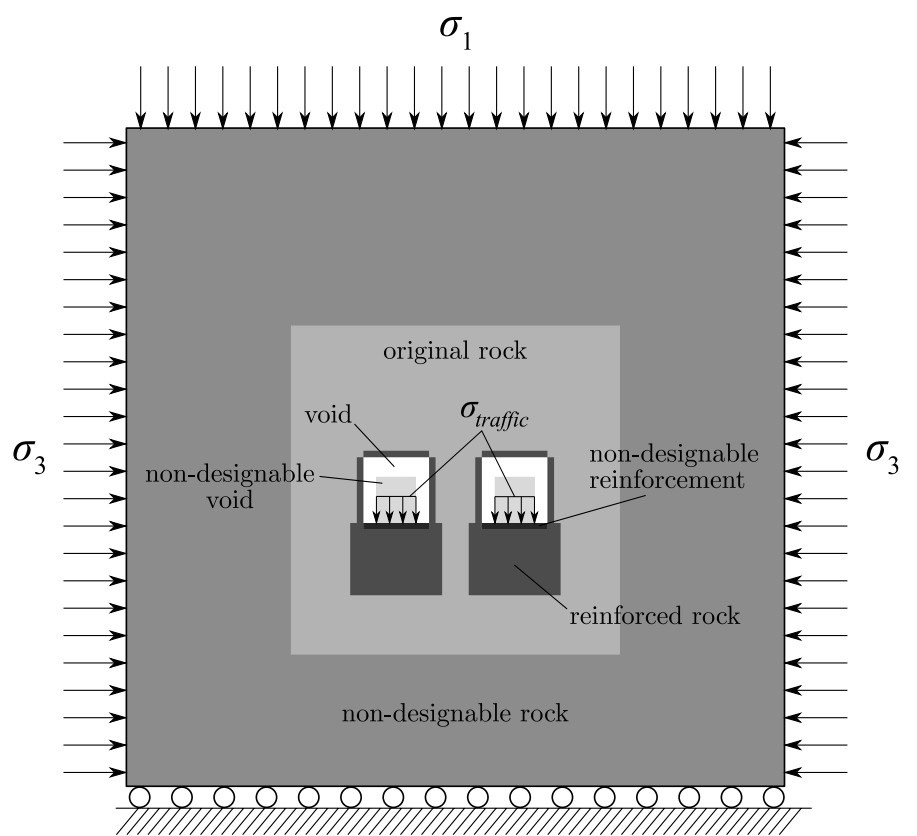

Fig. 8 An initial guess design illustrating the design domain, non-designable elements, loading, and restraints for two parallel tunnels.

two tunnels tend to join.
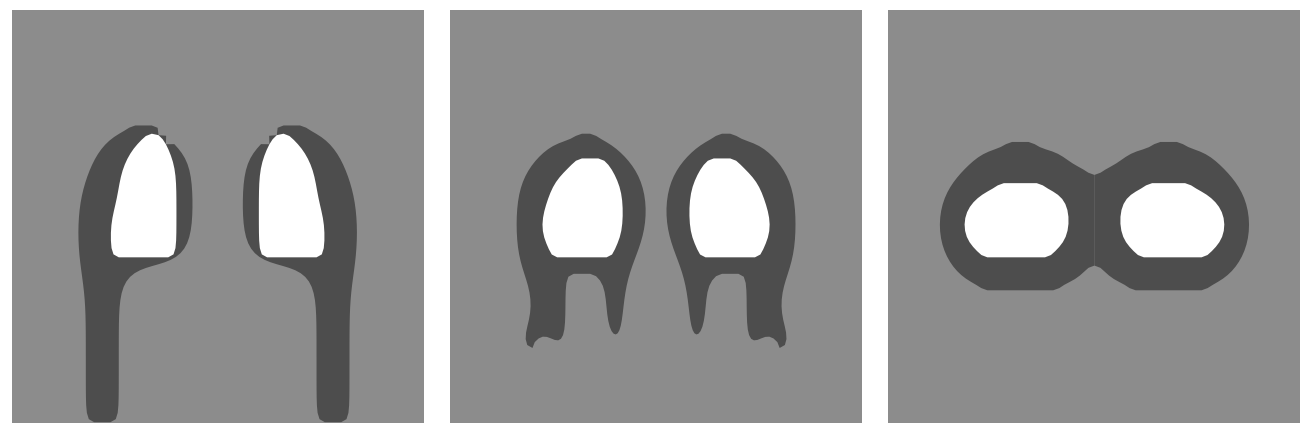

Fig. 9 The smoothed optimum designs for load ratios of $\lambda=0.4, \lambda=0.7$, and $\lambda=1.2$ (respectively from left to right) for the problem of two parallel tunnels in example 3.

\section{Conclusion}

The topology optimization of reinforcement around an underground opening in rock mass and shape optimization of the opening itself have been solved simultaneously. Among different topology/shape optimization methods available, the BESO method has been chosen due to its clear topology results and its fast convergence. However contrary to common BESO, in this paper a soft kill approach has been followed and a weak material has been used to model void elements. Mean compliance has been considered as the objective function for the optimization procedures together with constraints on maximum volume of reinforcements and on the size of the opening.

The problem is then reduced to two two-phase material distribution problems. The sensitivities of the objective function with respect to the design variables have been calculated for these problems. Two different sensitivity numbers have then been defined based on the calculated sensitivities. It has been shown that the two sensitivity numbers only differ in some constant coefficients. Hence the two optimization problems can be solved using nearly same computational effort as required by a single problem.

A shotcrete lining has been assumed around the opening with mechanical properties 
similar to that of reinforced rock. A filtering scheme has been used to prevent numerical instabilities such as checkerboard patterns. The filtering approach also smooths inter-material borders, resulting in a topology free of jagged edges.

To validate the method, some numerical examples have been solved. The evolution of the objective function shows a smooth, relatively monotonic and converging curve. A postprocessor has been used to smooth the boundaries of resulted topologies based on Bézier curves. The capability of the method has been tested by solving numerical examples with different loading conditions. The optimization of two parallel tunnels has also been addressed. It has been demonstrated via these examples that the extended BESO technique developed in this paper is capable of solving a range of topology and shape optimization problems in underground excavations.

\section{References}

( 1 ) Ren, G., Smith, J.V., Tang, J.W. and Xie, Y.M., Underground excavation shape optimization using an evolutionary procedure. Computers and Geotechnics, Vol.32,No.2 (2005), pp.122-132.

( 2 ) Ghabraie, K., Xie, Y.M. and Huang, X., Shape optimization of underground excavation using ESO method. In Innovations in Structural Engineering and Construction, Vol.2, Xie Y.M. and Patnaikuni I. (eds), (2008), pp.877-882, Taylor and Francis.

( 3 ) Liu, Y., Jin, F., Li, Q. and Zhou, S., A fixed-grid bidirectional evolutionary structural optimization method and its applications in tunnelling engineering. International Journal for Numerical Methods in Engineering, Vol.73,No.12 (2008), pp.1788-1810.

( 4 ) Yin, L., Yang, W. and Tianfu, G., Tunnel reinforcement via topology optimization. International Journal for Numerical and Analytical Methods in Geomechanics, Vol.24,No.2 (2000), pp.201-213.

( 5 ) Yin, L. and Yang, W., Topology optimization to prevent tunnel heaves under different stress biaxialities. International Journal for Numerical and Analytical Methods in Geomechanics, Vol.24,No.9 (2000), pp.783-792.

( 6 ) Yin, L. and Yang, W., Topology optimization for tunnel support in layered geological structures. International Journal for Numerical Methods in Engineering, Vol.47,No.12 (2000), pp.1983-1996.

( 7 ) Bendsøe, M.P., Optimal shape design as a material distribution problem. Structural Optimization, Vol.1,No.4 (1989), pp.193-202.

( 8 ) Bendsøe, M.P. and Kikuchi, N., Generating optimal topologies in structural design using a homogenization method. Computer Methods in Applied Mechanics and Engineering, Vol.71,No.2 (1988), pp.197-224.

( 9 ) Bendsøe, M.P. and Sigmund, O., Material interpolation schemes in topology optimization. Archive of Applied Mechanics, Vol.69,No.9-10 (1999), pp.635-654.

(10) Querin, O.M., Steven, G.P. and Xie, Y.M., Evolutionary structural optimisation (ESO) using a bidirectional algorithm. Engineering Computations, Vol.15,No.8 (1998), pp.1031-1048.

(11) Yang, X.Y., Xie, Y.M., Steven, G.P. and Querin, O.M., Bi-directional evolutionary method for stiffness optimization. AIAA Journal, Vol.37,No.11 (1999), pp.1483-1488.

(12) Xie, Y.M. and Steven, G.P., A simple evolutionary procedure for structural optimization. Computers $\mathcal{E}$ Structures, Vol.49,No.5 (1993), pp.885-896.

(13) Xie, Y.M. and Steven, G.P., Evolutionary structural optimization, (1997), Springer.

(14) Li, Q., Steven, G.P. and Xie, Y.M., On equivalence between stress criterion and stiffness criterion in evolutionary structural optimization. Structural Optimization, Vol.18,No.1 (1999), pp.67-73.

(15) Jing, L., A review of techniques, advances and outstanding issues in numerical modelling for rock mechanics and rock engineering. International Journal of Rock Mechanics and Mining Sciences, Vol.40,No.3 (2003), pp.283-353. 
(16) Brady, B.H.G and Brown, E.T., Rock Mechanics for Underground Mining, (2004), pp.200-201, Kluwer Academic Publishers.

(17) Ghabraie, K., Exploring Topology and Shape Optimisation Techniques in Underground Excavations, (2009), PhD Thesis, RMIT University.

(18) Bernaud, D., Debuhan, P. and Maghous, S., Numerical simulation of the convergence of a bolt-supported tunnel through a homogenization method. International Journal for Numerical and Analytical Methods in Geomechanics, Vol.19,No.4 (1995), pp.267-288.

(19) Bendsøe, M.P. and Sigmund, O., Topology Optimization - Theory, Methods and Applications, (2004), pp.16-18, Springer.

(20) Tanskanen, P., The evolutionary structural optimization method: theoretical aspects. Computer Methods in Applied Mechanics and Engineering, Vol.191,No.47-48 (2002), pp.5485-5498.

(21) Huang, X. and Xie, Y.M., Bi-directional evolutionary topology optimization of continuum structures with one or multiple materials. Computational Mechanics, Vol.43,No.3 (2008), pp.393-401.

(22) Sigmund, O. and Petersson, J., Numerical instabilities in topology optimization: A survey on procedures dealing with checkerboards, mesh-dependencies and local minima. Structural Optimization, Vol.16,No.1 (1998), pp.68-75.

(23) Li, Q., Steven, G.P. and Xie, Y.M., A simple checkerboard suppression algorithm for evolutionary structural optimization. Structural and Multidisciplinary Optimization, Vol.22,No.3 (2001), pp.230-239.

(24) Huang, X. and Xie, Y.M., Convergent and mesh-independent solutions for the bidirectional evolutionary structural optimization method. Finite Elements in Analysis and Design, Vol.43,No.14 (2007), pp.1039-1049. 